\title{
Predicting individual lung-function trajectories: An opportunity for prevention?
}

\author{
Milo A. Puhan MD PhD
}

See also www.cmaj.ca/lookup/doi/10.1503/cmaj.151483

$\mathrm{Z}$ afari and colleagues, in a linked paper, report on their model to predict lungfunction trajectories in individual patients. ${ }^{1}$ The model, which explains much of the variation in lung-function changes over time and performed well in validation cohorts, contributes knowledge beyond the findings of some recent cohort studies about the nature of lung-function changes over time. The prediction model may be useful for prevention and early treatment of chronic obstructive pulmonary disease (COPD).

In 1977, Fletcher and Peto reported on three findings in their famous article on lung-function changes over time based on a cohort of 792 men. ${ }^{2}$ They found substantial variability in the rate of change in lung function among smokers, former smokers and nonsmokers. Second, they reported that those with below-average lung function at study enrolment were more susceptible to developing COPD, whereas a substantial proportion of men with normal or above-average lung function did not develop obstructive lung disease despite continued smoking. Finally, they described smokers with respiratory symptoms but preserved lung function. It is unfortunate that, from Fletcher and Peto's article, it is mostly the simplified Figure 1, which emphasized a rather uniform accelerated decline of lung function in smokers, that became famous rather than the important findings described above.

Recent findings on lung-function trajectories as well as on symptoms and exacerbations associated with different levels of airway obstruction were similar to those of Fletcher and Peto despite many changes in environmental and behavioural exposures, as well as treatments. ${ }^{3-5}$ The prediction model by Zafari and colleagues ${ }^{1}$ goes beyond these findings because the authors found a smart way to combine predictors of the lung-function trajectory in individuals.

The development and validation of the prediction model was based on large cohort studies with diverse populations, which is ideal to develop widely applicable and robust prediction models. ${ }^{6}$ The predictive performance of the model is impressive, and the online tool provides four options (depending on the availability of predictors) to predict the forced expiratory volume at 1 second $\left(\mathrm{FEV}_{1}\right)$ trajectory together with an interpretation (e.g., rapid disease progression) and the probabilities of having a specific grade of severity as measured by the Global Initiative for Chronic Obstructive Lung Disease (GOLD) at each year over a follow-up period of 11 years. Thus three key features of prediction models to be used in practice are present - availability of predictors in real-world practice settings, robust evidence on external validity and an easy-to-use tool to obtain the prediction for an individual.

How can the model be used? The authors propose use of the model for both research and clinical practice. Prediction models can indeed be used to make clinical trials or observational studies more efficient and to identify subgroups of particular interest to study, for example, people with a predicted rapid decline in lung function in whom specific interventions may be beneficial to slow down the decline.

Zafari and colleagues argue that the prediction model for $\mathrm{FEV}_{1}$ provides opportunities for individualizing COPD prevention and management, for example, through risk-stratified treatment. Riskstratified treatment as a means of individualizing respiratory medicine has been proposed before. ${ }^{7}$ Risk-stratified approaches are attractive if a treatment is indicated only when the risk of the outcome to be prevented is higher than a certain threshold, because the treatment comes with harms or cost. Examples include low-dose acetylsalicylic acid

\section{KEY POINTS}

- A new prediction model explains much of the variability of individual lung-function trajectories; an online tool facilitates application of the model and offers different versions depending on the availability of predictors.

- This prediction tool can help individuals and health practitioners to identify the risk for a rapid decline in lung function and to motivate smoking cessation.

- Prediction tools may be useful in the prevention and early treatment of chronic obstructive pulmonary disease.
Competing interests: None declared.

This article was solicited and has not been peer reviewed.

Correspondence to: Milo Puhan, miloalan.puhan@uzh.ch

CMAJ 2016. DOI:10.1503 /cmaj.160611 
(ASA) and statins for primary cardiovascular prevention, and, in COPD, phosphodiesterase-4 inhibitors. These drugs have preventive effects (e.g., prevention of myocardial infarction by ASA and statins, and prevention of exacerbations of COPD by phosphodiesterase- 4 inhibitors, respectively) but come with potential adverse effects, which means that the benefits exceed the harms only in individuals at higher risk. ${ }^{89}$ Without valid prediction models, risk-stratified prevention or treatment is difficult to pursue; without them it is impossible to categorize patients accurately as being below or above a certain treatment threshold.

It seems uncertain whether the prediction model for $\mathrm{FEV}_{1}$ trajectories will be useful for riskstratified prevention or treatment of COPD. Advice and interventions for smoking cessation should be offered to any smokers irrespective of their risk of rapid decline in $\mathrm{FEV}_{1}$ and do not come with harms, so a risk-stratified approach may not be warranted. And for patients with diagnosed COPD, the clinical manifestations, including comorbidities and the risk for exacerbations and mortality, usually drive treatment decisions more than lung-function trajectories. The authors refer to a lack of prediction models for use in COPD and suggest that prediction models for exacerbations and mortality may now be developed following the example of their $\mathrm{FEV}_{1}$ prediction model. These statements are a bit surprising given that prediction models for patient-important outcomes, such as mortality and exacerbations, have been developed and widely discussed, and some have been validated in thousands of COPD patients. ${ }^{6,10}$

Instead, the prediction model by Zafari and colleagues provides new opportunities to identify individuals who are likely to progress to COPD or who are likely to have a rapid decline in lung function. The long-term perspective of the lung- function prediction model may motivate smokers to quit and thus may become an important component of individualizing smoking-cessation interventions and lead to higher quit rates. Another opportunity the prediction model offers is to explore whether the effects of some treatments differ in patients with a rapid decline versus patients with a slower decline as predicted by the model. If some treatments prove to be effective to slow down the decline in lung function in individuals at risk for rapid decline, the prediction model may be attractive to identify these individuals for early treatment.

\section{References}

1. Zafari Z, Sin DD, Postma DS, et al. Individualized prediction of lung function decline in chronic obstructive pulmonary disease. CMAJ 2016 Aug. 2 [Epub ahead of print].

2. Fletcher $\mathrm{C}$, Peto R. The natural history of chronic airflow obstruction. BMJ 1977;1:1645-8.

3. Vestbo J, Edwards LD, Scanlon PD, et al. Changes in forced expiratory volume in 1 second over time in COPD. N Engl J Med 2011;365:1184-92.

4. Lange P, Celli B, Agustí A, et al. Lung-function trajectories leading to chronic obstructive pulmonary disease. N Engl J Med 2015;373:111-22.

5. Woodruff PG, Barr RG, Bleecker E, et al. Clinical significance of symptoms in smokers with preserved pulmonary function. N Engl J Med 2016;374:1811-21

6. Puhan MA, Hansel NN, Sobradillo P, et al. Large-scale international validation of the ADO index in subjects with COPD: an individual subject data analysis of 10 cohorts. BMJ Open 2012;2:pii:e002152.

7. Agustí A, Antó JM, Auffray C, et al. Personalized respiratory medicine: exploring the horizon, addressing the issues. Summary of a BRN-AJRCCM workshop held in Barcelona on June 12, 2014. Am J Respir Crit Care Med 2015;191:391-401.

8. Yu T, Fain K, Boyd CM, et al. Benefits and harms of roflumilast in moderate to severe COPD. Thorax 2014;69:616-22.

9. Yu T, Vollenweider D, Varadhan R, et al. Puhan M a. Support of personalized medicine through risk-stratified treatment recommendations - an environmental scan of clinical practice guidelines. BMC Med 2013;11:7

10. Marin JM, Alfageme I, Almagro P, et al. Multicomponent indices to predict survival in COPD: the COCOMICS study. Eur Respir J 2013;42:323-32.

Affiliation: Epidemiology, Biostatistics and Prevention Institute, University of Zurich, Zurich, Switzerland

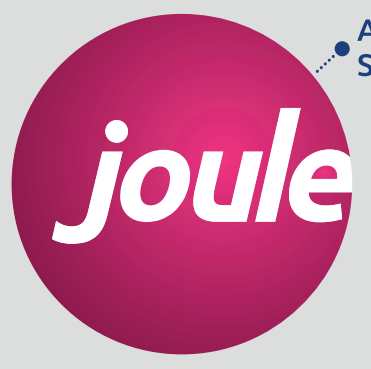
- they're developed.

Maximize your potential with courses from the Physician Leadership Institute.

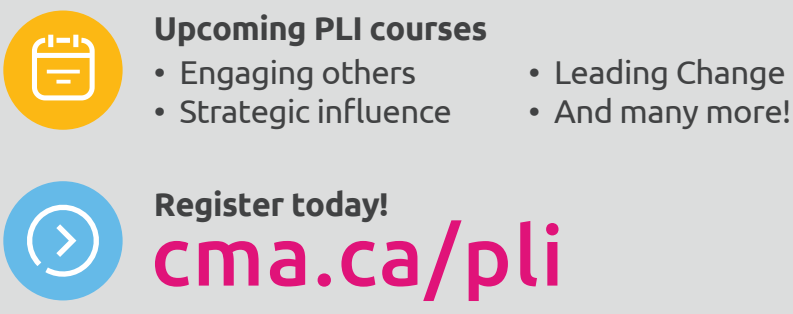

